\title{
The multi-variation approach
}

\author{
Cross-case analysis of ethnographic fieldwork
}

https://doi.org/10.1515/pjbr-2019-0017

Received September 26, 2018; accepted May 7, 2019

\begin{abstract}
This article argues that a multi-variation approach can be a useful supplement to existing ethnographic studies in the field of Human-Robot Interaction (HRI). The multi-variation approach builds on classical ethnographic case studies, where a researcher studies a delimited field in a microstudy of a particular robot, its makers, users, and affected stakeholders. The approach is also inspired by multi-sited studies, where researchers move across fields, adding to the complexity of the ethnographic findings. Whereas both approaches build on analysis of microstudies, the multi-variation approach is further inspired by postphenomenology, where the main aim is to deliberately seek variation - thus again adding to the complexity of the detailed findings. Here, the multivariation approach includes several researchers studying several types of robots across sites. The analytical approach seeks patterns across this complexity - and the claim is that a multi-variation approach has a strength in findings that are systematic and consistent across cases, sites, and variations. The article gives an example of such cross-variation findings in the robot field - namely the tendency for roboticists across cases and robot types to publicly present their robots as more finished and wellfunctioning than they actually are.
\end{abstract}

Keywords: ethnography, human-robot interaction, imaginaries, empirical studies, qualitative, anthropology, STS, human-technology relations

\section{Introduction}

In the field of Human-Robot Interaction (HRI), the term 'case studies' often refers to experimental set-ups created by the researchers themselves, where they test some

^Corresponding Author: Cathrine Hasse: Aarhus University, Danish School of Education, Copenhagen, Denmark;

E-mail: caha@edu.au.dk aspects of a human-robot interaction. In anthropology, ethnographic 'case studies' are typically delimited, but not entirely created, by the researchers themselves [14]. Such studies constitute bottom-up research using ethnographic methods, including analysis of observations, video-recordings, interviews, and field notes [5, 6]. These ethnographic micro-studies can go in depth into issues as they are "studying at first-hand what people do and say in particular contexts" [7, p. 4]. This article advocates a new methodology in HRI where analysis is not just made within singular cases or experimental settings but goes across several cases of ethnographic studies. Though this approach entails a loss of long-term embedded anthropological participant observations (which may result in a loss of refined ethnographic details), it opens up for a 'multi-variation approach' where analysis may find patterns across a multitude of bottom-up first hand studies of peoples' values, attitudes, and concerns tied to material artefacts.

\section{Ethnographic predicaments}

Case studies belong to the methodology of qualitative studies. "Qualitative research involves the studied use and collection of a variety of empirical materials - case study, personal experience, introspective, life story, interview, observational, historical, interactional and visual texts - that describe routine and problematic moments and meanings in individuals' lives," [8, p. 3].

Qualitative studies have been praised in recent years because they give insights to what people are saying and doing beyond assumptions. "Qualitative methods can by themselves produce compelling knowledge of how and why people behave as they do, whether in organisational, family, personal, or other social roles," [9, p. 2].

In qualitative research, ethnography is often the preferred approach even if it faces accusations of being narrow and too specific when contrasted with qualitative studies. As ethnographers, we aim at understanding local contexts and the local people's perspectives. As a result, 
however interesting, it is often difficult to make any wider claims based on ethnographic studies.

In anthropology, one of the disciplines that claims to build on ethnographic studies, ${ }^{1}$ the question of how to make local ethnographic research relevant on a larger scale has often been debated - not least in the so-called 'war' between the field of Science and Technology Studies and the natural and technical sciences [11, 12]. When social scientists study how scientific claims are made and constructed by cultural and social means, they run a risk of undermining their own position. Though ethnographers may get valuable insights through close up studies of people's everyday lives, these studies also beg some questions.

Anthropology does not always refer to case studies, though the case studies approach shares some predicaments with anthropology.

Where early anthropology was based on yearlong stays among native people (famously anthropologists like Bronislaw Malinowski, Margaret Mead, and Gregory Bateson) resulting in long monographies, much anthropology today seems to be close to a case study approach. Today the anthropological practice of ethnography is often reduced to short visits that last from a couple of days to a couple of months. At the same time 'ethno-graphy' - the written results of the ethnographic studies - has had a tendency to become more locally descriptive and less holistically analytical than the former monographies. Holism here refers to an encompassing analysis of the relation between people and the space they inhabit and (re)create. These changes have called forth issues of what status we should attribute this new kind of 'hit-and-run' ethnography in anthropology. Do we still gain insight into people's lives as a whole? Did we ever? As noted by the anthropologist Martyn Hammersley, we continue to expect ethnographic data gathered in particular places, in particular points of time to represent a general picture, even if we have gained no access to what people do when they are

\footnotetext{
1 Where anthropology is the discipline ethnography is often considered the methodology of anthropology. The anthropologist Tim Ingold (2011) is expanding this discussion as he points out that anthropology is what anthropologists 'live out' in the field whereas ethnography is what we choose to write about these experiences [10]. This is a novel debatable way to separate anthropology from ethnography - however in this context I stick to the understanding that ethnography is the way anthropologists and others gather data first-hand in an empirical field (which is the environment of everyday life for the ethnographic subjects), which is then put into words as analysis is formed. Often this process is taking place already in the field, and thus analysis and data gathering are inseparable.
}

not visited by the ethnographers [7]. Though new methodologies have emerged, like the multi-sited approach proposed by George Marcus, where people or things are followed around across local sites and acknowledging that locations are never isolated from a larger world system $[13,14]$, it still may render ethnographic analysis ahistorical, a-biographical in relation to particular participants and insensitive to larger patterns of change across space and time.

Anthropology most often has a critical dimension [15] that is not always found in non-anthropological case studies. This is most likely due to the inherently contrasting approach in anthropology, which more or less explicitly builds on what the anthropologist Laura Nader termed 'implicit comparisons' in cultural analysis [16, p. 84]. We tend to see how others differ culturally from ourselves as we learn that 'they' differ from how we view ourselves in a process of culture contrast. This often-implicit juxtaposition process can be made explicit and used as a research tool [17]. These comparisons imply a kind of translation $[18$, p. 9], but from an ethnographic point of view that also imply a process where an ethnographer learns to align with the ethnographic subjects and their (sometimes diverse) perceptions of material artefacts [19]. Criticism is increasingly from an insider position.

Whereas this process of learning with the field does not solve the ethnographic predicaments, it makes analytical approaches less plainly descriptive from an outside perspective. Yet, in-depth anthropological case studies still demand a long time in the field to obtain the perspective of insiders that make a critical positon from within a possibility (cf. [20, 21]).

\section{When anthropology goes big}

As a coordinator of several big anthropological projects building on ethnographic studies in several countries, I have often had to face this problem - and sometimes defend our research methodology from accusations of being merely based on local 'anecdotes'. My first big project in this respect was a project funded by the European Commission in 2005-2008 studying differences in university cultures across five European countries (Poland, Estonia, Italy, Finland, and Denmark). In each country, local researchers visited university departments of physics. Physics as a discipline is often considered to be 'outside' of cultural influence, but in Science and Technology Studies (STS) anthropological analysis have shown that culture and physics are interwoven even in relation to what 
constitutes scientific facts (e.g., [11]). These STS studies often build on directly contrasting one culture with another, as when Sharon Traweek contrasted the Japanese way of conducting particle physics with the American way [22]. Following this line of thinking, we constructed a model for studying university departments where we built on a method of 'culture-contrast' [23], holding findings from physics institutes in one country up against findings from physics institutes in four other countries. The local studies, however, were ethnographic studies as they were collections of notes from on-site visits and interview based statements on previous university careers and relations to colleagues. The focus was on human-human relations across disciplinary cultures. Other materials, such as knowledge about specific artefacts or values tied to agency and materials, were not included. This show that any large scale anthropological study necessarily already has a research ontology which sets limits for what can be studied.

In the UPGEM project (UPGEM stands for Understanding Puzzles in the Gendered European Map), 18 research assistants from Poland, Estonia, Denmark, Italy, and Finland made research at physics departments in their respective countries. This diversity added to the data analysis complexity, and eventually proved to be a strength. The culture contrast methodology built on the method of culture contrast, which was defined broader than a study of comparison - built on a 'tertium comparationis'. Though the assistants followed the same basic questionnaire, they were also asked to be open to surprises and present them in their reports.

The cross-cultural findings were not presented as 'case studies' - but had an overall objective of understanding the cultural diversity in the proportion of female physicists employed at universities across Europe. Like the few studies which have looked into this issue, the interviewbased studies of UPGEM also "found that the proportion of women employed as physicists differed from country to country" [24, p. 12]. However, through contrasting the local findings in the reports, we were able to identify aspects present in one national context which we next could identify as absent in the other national reports. This led to an identification of patterns across the huge data material of physics as culture with intrinsic values found across national differences - and physics in culture informed by national histories and values. By using the culture contrast method in this way, new self-evident connections (which might otherwise not be noticed by the researcher) were revealed across the set of reports, which gave new insights into why we found differences in female career paths from country to country. An example of this was the finding (which had seemed self-evident to Italian researchers) of the importance of accepting students with a background in classical studies (Latin and Greek) in Italy, whereas this possibility was out of the question in Denmark [17]. Thus, in spite of physics being considered outside of culture [22], with an anthropological approach contrasting the individual reports across national cultures we could confirm, like Traweek, that culture matters for people's lives and careers - even in physics [24].

(The lessons learned from this big scale project was enhanced and refined in a subsequent study of technological literacy among teachers and nurses in Denmark (2011-2015) where teachers' technological literacy was contrasted with nurses' and engineers' $[25,26]$.)

\section{Towards a new type of case methodology}

In the ongoing REELER (Responsible and Ethical Learning with Robotics) project (2017-2020), we have materiality as the pivotal point: the robot. Robots can be defined in many ways, for instance as

\begin{abstract}
"capable of performing tasks by sensing its environment and/or interacting with external sources and adapting its behaviour. As examples, the standard gives an industrial robot with a vision sensor for picking up and positioning an object, mobile robots with collision avoidance and legged robots walking over uneven terrain.” $[27$, p. 10]
\end{abstract}

After a review of the concept in social science and technical literature, we decided to take a point of departure in the ISO standard 8373:2012 definition:

A robot is an actuated mechanism programmable in two or more
axes with a degree of autonomy, moving within its environment,
to perform intended tasks. Autonomy in this context means the
ability to perform intended tasks based on current state and
sensing, without human intervention.

We also decided to let the concept develop as we studied what robot designers across Europe themselves defined as robots. So when we prepared the groundwork for the project, the concept robot was included in almost all of our database searches as it frames all of our other concepts in the project, including the methodological concept of "case study'. From these first searches (systematic EPPI-inspired database searches), it appears that studying robots as case studies across contexts is a novelty. As noted in a report by REELER assistant Jessica Sorenson: 


\begin{abstract}
"A search of SCOPUS for robot* began with 3 hits in 1924, following the publication of Čapek's R.U.R., which coined the term. There was very little peer-reviewed literature mentioning robot until the 1980s. In the 1980s and 90s, there was a steady rise in peer-reviewed publications. Beginning in the year 2000, there was an exponential growth in mention, from under 5k texts per year to almost $25 \mathrm{k}$ texts per year.” [28, p. 2]
\end{abstract}

However, when these references were connected to case studies the picture was as mentioned in the introduction to this contribution: Cases were largely creased by the social scientists as experiments or microstudies of a primarily local character. These studies are not the holistic anthropological studies of the past that included analysis of a larger context such as a cosmology shared by a local group of people. Hammersley has characterized this kind of studies as connected to the new possibilities for using electronic research equipment:

"[P]artly as a result of the increasing use of audio- and videorecording devices, there has been a growing tendency for ethnographers to carry out detailed micro analysis of what was actually said and done on particular occasions.” $[7$, p. 6]

As REELER has the general purpose of identifying and aligning gaps in how robot makers learn about people affected by their robotic creations (beyond the envisioned users), we had to think of novel ways to move beyond the close-up microstudies of robot makers testing their machines on their own choice of users. What we needed were a number of cases that could enlighten us about the many different stakeholders to be affected by robots in the future, and next to find ways to make the robot makers themselves learn about the effects of their work. The idea of making 10 separate ethnographic microstudies in robot makers' laboratories and study how they thought about users in each case was tempting, but left us with the basic question of how these cases could relate to each other. If the holistic part of the case study was not included, we ran the risk of presenting very local findings as general statements without even considering how local the studies were. If, on the other hand, we made in-depth holistic studies of ethnographic laboratories, we ran the risk of not having the time to also identify and involve the affected stakeholders we wanted the roboticists to learn about. If the robot makers were to learn to be more ethical through our research findings, by including an expanded understanding of affected stakeholders than the immediately envisioned users of robots, we had to find a way to solve this conundrum. We decided to make ten studies seeking variation in robot types spanning from industrial robots, over service robots to social robots. Here Kate Darling notes an important distinction between industrial and service robots which are not meant to be social, and social robots:

\begin{abstract}
"A social robot is a physically embodied, autonomous agent that communicates and interacts with humans on an emotional level. For the purposes of this article, it is important to distinguish social robots from inanimate computers, as well as from industrial or service robots that are not designed to elicit human feelings and mimic social cues. Social robots also follow social behavior patterns, have various 'states of mind', and adapt to what they learn through their interactions." [40, p. 4]
\end{abstract}

We were well aware of the huge differences between types of robots - which all come with their own kind of special ethical issues. One of those differences is between social robots and industrial robots. Apart from the differences between industrial robots and social robots noted by Darling, we also found it to be an important difference that industrial robots were often 'caged' in spaces especially designed for them, whereas social robots have to either adapt to existing human environments or need environments to be changed adapt to the social robots. Ethical issues change when the robots move right into people's homes. However, in spite of huge differences between robot types REELER found a number of ethical issues, which were found across cases i.e. where the same kind of ethical issue is found distributed across the robot type. We found examples where industrial robots were 'sold' in the media as autonomous and intelligent when in fact they demanded a lot of maintenance. We found industrial robots being compared to dancers, and social just like the industrial robots were presented as more capable in media versions. This 'overselling' of robot capabilities in media - and our field experiences of robots that did not live up to the media presentations - went across field studies.

At first glance, we could be seen as conducting a kind of multi-sited ethnography, where the practice of ethnographic fieldwork is pursued in more than one geographical locations. Though multi-sited is a helpful concept [13], it is important to make clear that although anthropologists may have imagined 'the field' to be one location as part of the anthropological discipline's research, anthropology has in a way always been 'multi-sited'. As already noted, Laura Nader found the multi-sited juxtaposition to be an inherent (albeit often implicit) aspect of all ethnographic fieldwork. When ethnographers cannot help contrasting what they know from their of background with a new culture - as when, for instance, white feminist American ethnographers studied the role of women in Muslim countries [16]. Even the old holistic monographies show that anthropologists always moved between a 'home' site and a new site. Even when staying in one national place, 
Table 1: Example of REELER case variation.

\begin{tabular}{c|c|c|c|c|c}
\hline Robot sector & Agriculture & Health & Education & Industry & Construction \\
\hline Specific robot & $\mathrm{Xi}$ & $\mathrm{Yi}$ & $\mathrm{Zi}$ & $\mathrm{Ni}$ & $\mathrm{Ti}$ \\
\hline Specific PI location & Italy & Poland & Denmark & Germany & Spain \\
\hline Users & Sweden & Poland & Denmark & Germany & Spain \\
\hline Affected stakeholders & Spain & Denmark & Belgium & Estonia & UK \\
\hline
\end{tabular}

like 'fieldwork at home', anthropologists always carried their own perspectives from their own background with them. This is the implicit comparison Laura Nader identified - and that we try to make explicit in our REELER work.

In REELER, the researchers come from Poland, Denmark, Germany, United States, UK, Italy, the Netherlands - and our research focus is on European robotics, which we soon found to be entangled with robotics in Japan and United States. Our research sites include robotic laboratories in more than 15 European countries implicated in all kinds of trade arrangements with other countries all over the globe (see Figure 1). When we (in REELER) claim to be working multi-sitedly, we acknowledge that ethnographic research is inherently embedded in a world system. Multisited ethnography is a fieldwork methodology emergent out of heterogeneity and globalization. Such research implies moving away from conventional ethnographic approaches that rely on single and local sites towards "the circulation of cultural meanings, objects and identities in diffuse space-time” [13, p. 96]. The goal is to investigate not only variously situated robots dispersed across Europe but also to find analytical connections among such sites. Furthermore, we expand and make use of the notion of multi-sited by going beyond what the robot makers in local laboratories themselves expect to be 'users' by identifying other subjects likely to be affected by the robots they create. We have named these 'affected stakeholders' - and ask questions about how the roboticists may or may not include considerations of affected stakeholders in their design.

In summary, we acknowledge that as researchers we are always implicitly comparative - and that whatever we follow in the world system is an effect of our own previous learning processes. That is why the main objective of REELER is not a fixed analytical object, but moves as we learn to change our own perspectives on the robotic world.

\section{A new variation-based multi-sited fieldwork}

Following our experiences from earlier projects, we have tried to benefit from the idea of looking across a multitude of perspectives in REELER. Instead of viewing it as a weakness that we work on very different robots, being created in very different locations, with very different envisioned users, and different affected stakeholders as well, we now look at this 'messy' amalgamation as a strength.

First a few words on how we work. In REELER, we have decided to make use of variation as a research strategy. Our research is confined to robots which are primarily made in Europe, but the robots we look at are all examples of 'robot types' which we have decided to specify from a number of variables (e.g., countries and robot types; see Table 1).

The robot names in Table 1 are made up - and the cases presented here are not real cases as we work under strict non-disclosure agreements with the companies we work with. All the robots chosen have a PI = a main project manager, but also have networks and collaborative robot maker partners all over Europe, in US, and as far as Japan. Thus, no robot is being built in one location - a robot is a distributed technology.

Our work on robot types can be illustrated with the following example. We have made ten case studies and in each case, we take our point of departure in a type of robot - for instance, a construction robot, $T i$, from Spain created to help build brick houses. The users are in this case identified as 'brick workers' - and a few from the local environment in the local town of the PI are called in to test the brick laying robot. We create a case around this robot (e.g. Ti from Spain) and visit the robotics laboratories in Spain but also the partners the PI collaborate with in Germany, Italy, Sweden, Denmark, and the UK. These collaborators for instance develop the mobile base, user interface, or guidance system to be incorporated into the robotic system in Spain, where testing occurs. Thus, in a single case we may have up to 6-7 different visits and interviews with robot makers from all over Europe. Of course, we cannot explore all of the sites that contribute to the design of the robot in question for reasons of limited funding and time. Therefore, we get limited knowledge of each robot type, and even limited knowledge of the particular robot we have chosen to represent a particular robot type. In identifying affected stakeholders, we often include the users envisioned by the robot makers themselves (in this case local construction workers), but then we move to other people not tied to the project that we have identified 
during fieldwork as affected stakeholders. These may include:

- People who educate construction workers - how much have they taken this new technology into account? How will it affect their work in the future?

- Unions organizing construction workers. How much have they considered this new technology? How will it affect their work in the future?

- Construction workers from other countries who may have other traditions and values tied to work.

In this way, we get 10 thorough cases that are written up as reports with a review of the robot type, descriptions of the history of the design of particular construction robot, how collaboration between partners evolved and involved users through sites and meetings - and interviews with as many as 12-25 people in a case. For an ethnographic study, 10 case studies made this way is an enormous amount of rich data material - and notoriously difficult to work with from the traditional anthropological methods of holistic analysis. The cases, however, are not just microstudies for comparison. They present us with huge variation where each case has some very specific issues tied to:

- The type of robots (an educational robot differ from a construction robot in particulars ways we can specify);

- The robot makers (informed by different funding schemes and national agendas);

- The users defined by the roboticists (workers and teachers differ from country to country);

- The affected stakeholders (teachers differ from union workers and construction workers).

What we have done is to look carefully at each case write-up and identify all of the issues relevant for how roboticists can make more ethically designed robots that come up in each case. Next, we have made a cross-case variation analysis through NVivo qualitative data analysis software, coding of all interview material. In all of this work, we at first deliberately seek variation - and do not work from a preselected tertium comparationis. This approach (according to our database searches) has not before been conducted anywhere in the field of HRI. It is inspired from phenomenology where the variation approach began with a Husserlian philosophical examination - for instance a human looking at an apple from many different angles to determine its essence:

"Husserl's earlier use, variations (originally derived from mathematical variation theory) were needed to determine essential

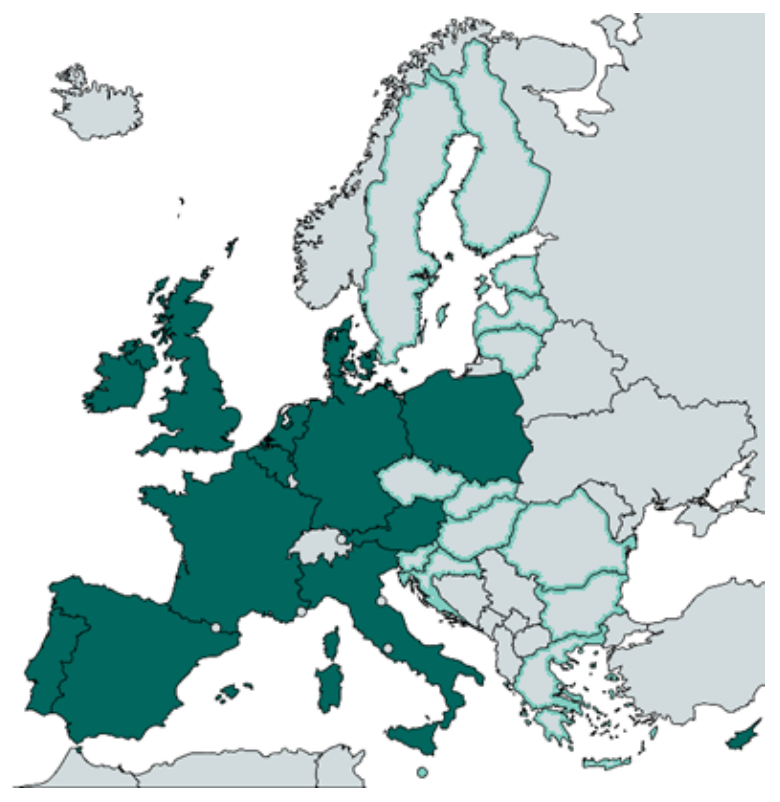

Figure 1: REELER case variation across Europe.

structures, or 'essences.' Variations could be used to determine what was variant, and what invariant.” [29, p. 12]

In the new versions of postphenomenology this search for essence has for long been abandoned (e.g., [30]). The method of finding patterns across variations however is still useful. The techno-philosopher Don Ihde has expanded on the method to include how apparently 'same' objects are taking on different meaning in practice, where moving across time and space has expanded the objects' meaning:

"I am not claiming here to have exhausted the variations, but these [examples] are enough to show that the phenomenological variations which now include considerations of the materiality of the technologies, the bodily techniques of use, and the cultural context of the practice, are all taken into account and demonstrate again the importance of variation theory with its outcome in multistability, the role of embodiment, now in trained practice, and the appearance of differently structured lifeworlds relative to historical cultures and environments." [29, pp. 18-19]

Inspired by postphenomenology and other sources of socio-cultural theory from the field of STS, we began to look for patterns across variation in and across cases and we find that the variation and the complexity of the rich dataset to be strengths of the methodology. We now make the claim that if we were to find patterns across all cases, these patterns emerge in spite of the huge variations, thus giving credit to the importance of our findings. In the preliminary cross-case variation analysis, we have indeed 
identified some themes that go across cases - even if each case is also rich with case-specific material. One example of this is that robot makers (across countries, robot types, and laboratory collaborations), when they present their machines to local users and to each other, have a tendency to present their unfinished robot designs as more 'finished' and well-functioning than is actually the case. We've seen this at conferences, in showrooms, and online - and contrasted these public representations with the ethnographic observations made in REELER cases. For example, a building robot, a harvesting robot, and an educational robot have been shown working side-by-side with humans in public videos (these are just examples, as we cannot disclose the actual cases studied) - whereas in all of these cases we've discovered that the robots in practice are far from working smoothly with humans. This tendency to enhance robots' ability in public media could well reinforce what the robot makers themselves have termed the 'Hollywood effect', where humans come to expect robots to be like the ones they encounter in movies [31, p. 56]. For affected stakeholders, this means they may hold expectations for robots that are higher than what real robots can actually live up to. This is a kind of robot innovation 'bubble' that we may expect in the future, as we now have many (also published) examples of how robot technologies often disappoint when implemented in people's everyday practices - and especially when the robots are expected to work closely in environments not tailored to human-robot interactions.

\section{Multi-sited and multi-variation}

The multi-variation approach both draws on and differs from a multi-sited approach. As discussed by MarkAnthony Falzon and his colleagues in the anthology Multisited Ethnography: Theory, Praxis and Locality in Contemporary Research [32], the term multi-sited has many, sometimes contradictory, definitions. Multi-sited ethnography, however, as a whole breaks with the convention that ethnography takes place in one site where the ethnographers learn about the local people by living with them over a considerable period, and considers their local existence contextualised by a global world system. The multisited approach proposed by George Marcus in 1995 in Annual Review of Anthropology broke with these conventions. The 'world-system' would no longer be seen as outside or around the local site but an inherent feature of social phenomena that could not be explored in one site alone, but only by following people, things, metaphors, stories "across space (because they are substantially continuous but spatially non-contiguous). Research design proceeds by a series of juxtapositions in which the global is collapsed into and made an integral part of parallel, related local situations, rather than something monolithic or external to them," [32, p. 1].

Like the multi-sited approach in terms of methods, the multi-variation approach also uses traditional methods like interviews, note-taking, etc. However, where the multi-sited approach "involves a spatially dispersed field through which the ethnographer moves - actually, via sojourns in two or more places, or conceptually, by means of techniques of juxtaposition of data” [32, p. 2], the multivariation approach does not aim at one ethnographer following people, things, \& stories across spaces. Instead, the approach involves juxtapose data collected by several researchers exploring different sites looking for variation within each case, just as the cases themselves are chosen as variations upon a common theme: in our REELER example, the design of robots.

The variation is sought for deliberately finding complexity within each case and across cases in order to make the final claim, that if some coherent relevant patterns are found across such complexity and variation, it is no longer important if each case is 'local' as the variation shows a pattern that only emerges in analysis across all of these local-global sites.

Like Ihde, we do not claim to have exhausted the variations. Therefore, our findings of patterns could also change if the number of variations were enhanced (or diminished). The phenomenological variations of robot designs and user involvements are tied to "the materiality of the technologies, the bodily techniques of use, and the cultural context of the practice" [29, p. 18]. Across this multistability, we find patterns that tie the disparate robotic lifeworlds together. However, the patterns found are most likely also both relative to history, as they point to common cultural values tied to the practices of robotic design.

Apart from the tendency to exaggerate the performance of the robots developed, we found a number of other issues across sites, such as unreflected aspects of who was included and excluded by the robots designed (e.g. taking a prototypical male size hand for robot operating systems laying bricks), or assuming a robot designed to be universal, where our research showed the robot was suitable for use only in sites close to the home environments where the robots were designed (thus ignoring differences in physical environments and/or differences in Northern and Southern European healthcare or worker policies, e.g.). 


\section{Conclusion}

As the REELER project is ongoing, it is not possible at this stage to go more into details with our cross-case variation analysis. At this stage, we may just point the attention to the possibility of how doing ethnographic work in HRI can also work in large-scale ethnographic designs. Ethnographic case studies differ from many of the case studies presented in the field of HRI in two ways:

1) the researchers do not set up the case to be studied themselves. Even if ethnographers always influence the framing of what they study, they only rarely choose to create a new reality which they then study as a case. They are much more interested in finding out how human-robot interactions play out in everyday situations.

2) Though existing HRI studies are inherently multi-sited, the researchers are not always aware of this and sometimes make very close-up microstudies. However, in an anthropological understanding of ethnographic case studies there is always a search for a larger context. This context can include people and things present at the locations, but may also move beyond the setting.

In a multi-sited approach, the world-system is inherent in local sites. However, in the multi-variation approach, the patterns that go across the cases have to be elicited analytically. It is not so much a world-system as a pattern of values and issues that goes across even highly varied local case studies. The new methodology of finding cross-case patterns across a multitude of variations is a possibility for ethnographers to enhance the claims that can be made from ethnographic case studies. In that respect, the multi-variation approach indicates a new and important way to use ethnographic studies in HRI.

\section{References}

[1] W.-Y. Hwang, S.-Y Wu, A case study of collaboration with multirobots and its effect on children's interaction, Interactive Learning Environments, 2014, 22(4), 429-443

[2] L. Major, T. Kyriacou, P. Brereton, The effectiveness of simulated robots for supporting the learning of introductory programming: a multi-case case study, Computer Science Education, 2014, 24(2-3), 193-228

[3] A. Ioannou, E. Andreou, M. Christofi, Pre-schoolers' interest and caring behaviour around a humanoid robot, TechTrends, 2015, 59(2), 23-26

[4] S. Somyürek, An effective educational tool: Construction kits for fun and meaningful learning, International Journal of Technology and Design Education, 2015, 25(1), 25-41

[5] M. C. Ayar, First-hand experience with engineering design and career interest in engineering: An informal STEM education case study, Educational Sciences: Theory \& Practice, 2015, 15(6), 1655-1675

[6] O. G. Stewart, M. E. Jordan, "Some explanation here": a case study of learning opportunities and tensions in an informal science learning environment, Instructional Science, 2017, 45(2), 137-156

[7] M. Hammersley, Ethnography: Problems and prospects, Ethnography and Education, 2006, 1(1), 3-14

[8] N. K. Denzin, Y. S. Lincoln, Introduction: Entering the field of qualitative research, In: N. K. Denzin, Y. S. Lincoln (Eds.), The Sage Handbook of Qualitative Research, Sage, 1994, 1-17

[9] J. Hogan, P. Dolan, P. Donnelly, Introduction, In: J. Hogan, P. Dolan, P. Donnelly (Eds.), Approaches to Qualitative Research: Theory and Its Practical Application, Oak Tree Press, 2009, 1-18

[10] T. Ingold, Being alive: Essays on Movement, Knowledge and Description, Routledge, 2011

[11] S. Sismondo, An Introduction to Science and Technology Studies, Wiley-Blackwell, 2010

[12] E. J. Hackett, O. Amsterdamska, M. Lynch, J. Wajcman, The Handbook of Science and Technology Studies, The MIT Press, 2008

[13] G. E. Marcus, Ethnography in/of the world system: The emergence of multi-sited ethnography, Annual Review of Anthropology, 1995, 24, 95-117

[14] G. E. Marcus, Multi-sited ethnography: Five or six things I know about it now, Problems and Possibilities in Multi-sited Ethnography [workshop], University of Sussex, 27-28 June 2005, http: //eprints.ncrm.ac.uk/64/1/georgemarcus.pdf

[15] E. F. Fischer, Cultural logic and Maya identity: Rethinking constructivism and essentialism, Current Anthropology, 1999, 40(4), 473-500

[16] L. Nader, Comparative consciousness, In: R. Borofsky (Ed.), Assessing Cultural Anthropology, McGraw-Hill, 1994

[17] C. Hasse, S. Trentemøller, The method of culture contrast, Qualitative Research in Psychology, 2009, 6(1-2), 46-66

[18] A. Gingrich, R. G. Fox (Eds.), Anthropology, By Comparison, Routledge, 2002

[19] C. Hasse, An Anthropology of Learning, Springer Verlag, 2015

[20] J. Sorenson, Decisions and values: Engineering design as a pragmatic and sociomaterial negotiation process, REELER Working Paper Series [working paper], Aarhus University, 2018

[21] S. Hansen, If we had a specific idea of the product 12 months ago, it would never be what we have today! A study in situational pragmatic actions and strategies in everyday technological development, REELER Working Paper Series [working paper], Aarhus University, 2018

[22] S. Traweek, Beamtimes and Lifetimes: The World of High Energy Physicists, Harvard University Press, 1988

[23] C. Hasse, S. Trentemøller, Cultural work place patterns in academia, Science Studies 2011, 24(1), 6-23

[24] C. Hasse, S. Trentemøller, Break the Pattern. A Critical Enquiry into Three Scientific Workplace Cultures: Hercules, Caretakers and Worker Bees, Tartu University Press, 2008

[25] C. Hasse, Technological literacy for teachers, Oxford Review of Education, 2017, 43(3), 365-378

[26] J. Wallace, C. Hasse, Situating technological literacy in the workplace, In: J. Dakers (Ed.), New Frontiers in Technological Literacy, Palgrave Macmillan, 2014, 153-164

[27] N. Nevejans, Study: European civil law rules in robotics, Policy Department for Citizens' Rights and Constitutional Affairs, European Parliament. Document PE 571.379, 2016, 
http://www.europarl.europa.eu/committees/fr/supportinganalyses-search.html

[28] J. Sorenson, SEARCH REPORT: Education and Robot [internal REELER project document], 2017

[29] D. Ihde, Postphenomenology and Technoscience: The Peking University Lectures, SUNY Press, 2009

[30] J. Aagaard, J. K. B. Friis, J. Sorenson, O. A. Tafdrup, C. Hasse (Eds.), Postphenomenological Methodologies: New Ways in Mediating Techno-Human Relationships, Rowan \& Littlefield International, 2018

[31] KUKA, Kuka Annual Report 2016, Making the Right Decisions at the Right Time, 2016

[32] M.-A. Falzon, Introduction, In: M.-A. Falzon (Ed.), Multi-sited Ethnography: Theory, Praxis and Locality in Contemporary Research, Ashgate, 2009, 1-23

\section{See also:}

[33] L. Blond, Technological style is history, SHOT Graduate Workshop Paper [paper presentation] presented at SHOT Annual Conference, 2016 Singapore [working paper], 2016, 1-9

[34] M. Bruun, C. Hasse, S. Hanghøj, Studying social robots in practiced places, Techne: Research in Philosophy and Technology, 2015, 19(2), 143-165

[35] K. M. Eisenhardt, M. E. Graebner, Theory building from cases: Opportunities and challenges, Academy of Management Journal, 2007, 50(1), 25-32

[36] K. M. Eisenhardt, Building theories from case study research, Academy of Management Review, 1989, 14(4), 532-550

[37] J. Gerring, The case study: what it is and what it does, In: C. Boix, S. C. Stokes (Eds.), The Oxford Handbook of Comparative Politics, Oxford University Press, 2007, 94-109

[38] J. Gerring, Case Study Research: Principles and Practices, Cambridge University Press, 2006

[39] R. K. Yin, Case Study Research: Design and Methods, Sage, $1994 / 2003$

[40] K. Darling, Extending legal rights to social robots [paper presentation], presented at We Robot Conference, University of Miami, 23 April 2012, http://dx.doi.org/10.2139/ssrn.2044797 (accessed April 26, 2019) 